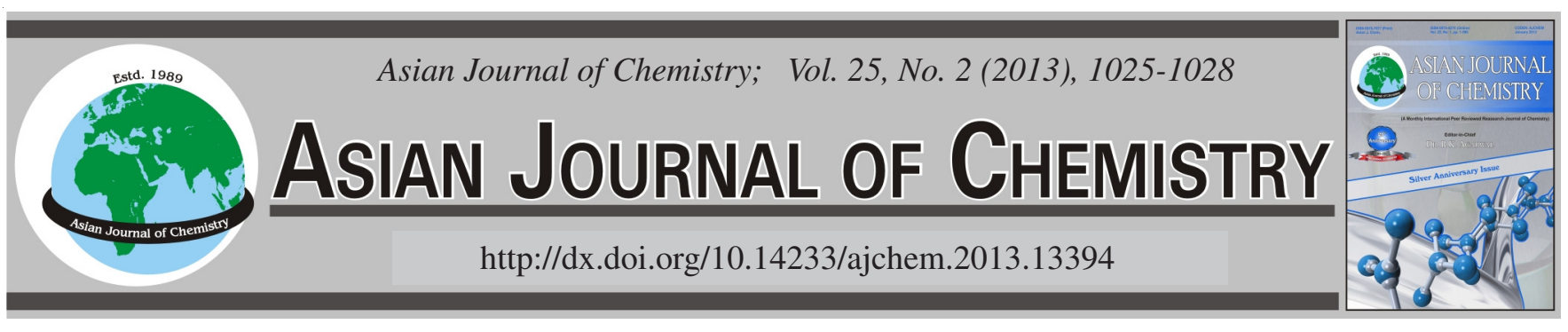

\title{
Synthesis of Super Absorbent Hydrogels with Using Releasing Drug
}

\section{Fatemeh Soleimani ${ }^{1, *}$, Mohammad Sadeghi ${ }^{2}$, Hadis Shasevari ${ }^{1}$, Arezoo Soleimani $^{1}$ and Hossein Sadeghi $^{3}$}

\author{
${ }^{1}$ Young Researchers Club, Khorramabad Branch, Islamic Azad University, Khorramabd, Iran \\ ${ }^{2}$ Department of Chemistry, Science Faculty, Arak Branch, Islamic Azad University, Arak, Iran \\ ${ }^{3}$ Department of Chemistry, Science Faculty, Khorramabad Branch, Islamic Azad University, Khorramabad, Iran
}

*Corresponding author: Fax: +98 861 3670017; Tel: +98 916 1613256; E-mail: fatisoleymani@yahoo.com

\begin{abstract}
In this work, a novel family of $\mathrm{pH}$-responsive polymeric hydrogel based on collagen was prepared for realesing drug. Acrylic monomers, acrylic acid and itaconic acid were simultaneously graft copolymerized onto collagen backbones by a free radical polymerization technique using ammonium persulfate as initiator and methylene bisacrylamide as a crosslinker. Hydrogel formation was confirmed by FTIR spectroscopy. Thermogravimetric analysis showed the thermal stabilities of the hydrogels. Results from scanning electron microscopy also showed a porous structure with smooth surface morphology of the hydrogel the super absorbent hydrogels exhibited high sensitivity to $\mathrm{pH}$, so that, several swelling changes of the hydrogel were observed in $\mathrm{pH}$ variations of a wide $\mathrm{pH}$ range (1-13).
\end{abstract}

Key Words: Collagen, Hydrogel, Acrylic acid, Itaconic acid, Controlled release.

\section{INTRODUCTION}

Drug delivery systems (DDSs) are regarded as a promising means to control post-operative inflammation ${ }^{1}$, although design improvements are needed to increase biocompatibility and effectiveness, as well to prolong controlled release of the $\mathrm{drug}^{2}$. Interest in biodegradable polymers and specifically in a drug delivery system matrix, has been growing. The main reason for this is that delivery systems based on biodegradable polymers do not require removal of the polymers from the body at the end of the treatment period, as they degrade into physiologically occurring compounds that can be readily excreted from the body ${ }^{3}$.

In recent years, much interest has been shown in the development of synthesis of natural-based super absorbent hydrogels $s^{4-5}$. These biopolymer materials are crosslinked hydrophilic polymers, capable of absorbing large quantities of water, saline or physiological solutions ${ }^{4}$. Because of their non-toxicity, biocompatibility and biodegradability, naturalbased hydrogels have attracted in many fields such as hygienic, cosmetics and agriculture ${ }^{6}$.

Stimuli-responsive smart hydrogels that can respond to environmental physical and chemical stimuli, such as temperature, $\mathrm{pH}$, light, electric field and magnetic field have attracted great interests in recent years due to their versatile applications such as controlled drug and gene delivery systems ${ }^{6}$, chemical/bio-separations and sensors and/or actuators. Among those smart hydrogels, pH-responsive hydrogels have been extensively investigated for potential use in site-specific delivery of drugs to specific regions of the gastrointestinal tract and have been prepared for delivery of low molecular weight drugs.

Proteins are widely distributed in nature and are synthesized mainly in animals, i.e., collagen and keratin etc. and in a few plants such as soya. In general, proteins are high molecular weight polymers and their solubility in aqueous solutions is difficult. Two efficient methods for preparation of aqueous soluble proteins are alkaline and enzymatic hydrolysis. According to the literature survey, a few studies have been reported in the case of protein-based hydrogels ${ }^{7}$. Hence, the target of the current study was to exploit novel $\mathrm{pH}$-sensitive collagenbased hydrogels for the effective controlled releaseing drug system. The absorption of drug and release capacities of hydrogel systems were also examined.

\section{EXPERIMENTAL}

Hydrolyzed collagen (Parvar Novin-E Tehran Co.) was industrial grade which is available in market and has nearly $25 \%$ insoluble phosphate salt. Acrylic acid (AA, Merck) was used after vacuum distillation. Itaconic acid (IA, Merck) and ammonium persulfate (APS, Merck) were used without purification. Methylene bisacrylamide (MBA, Fluka) was used as received. All other chemicals were of analytical grade. Double distilled water was used for the hydrogel preparation and swelling measurements. 
Preparation of hydrogel: A general procedure for chemically crosslinking graft copolymerization of acrylic acid and itaconic acid onto collagen backbones was conducted as follows. Hydrolyzed collagen (1.33 g) was dissolved in $50 \mathrm{~mL}$ distilled water and filtered to remove its insoluble phosphate salt. Then the solution was added to a three-neck reactor equipped with a mechanical stirrer (Heidolph RZR 2021, three blade propeller type, $300 \mathrm{rpm}$ ). The reactor was immersed in a thermostated water bath preset at a desired temperature $\left(80^{\circ} \mathrm{C}\right)$. Then a definite amount of ammonium persulfate solution $(0.1 \mathrm{~g}$ in $5 \mathrm{~mL} \mathrm{H}_{2} \mathrm{O}$ ) was added to collagen solution and was allowed to stir for $10 \mathrm{~min}$. After adding ammonium persulfate, certain amounts of acrylic acid (1.20 g) and itaconic acid (0.80) were added simultaneously to the collagen solution. Methylene bisacrylamide solution $\left(0.05 \mathrm{~g}\right.$ in $\left.5 \mathrm{~mL} \mathrm{H}_{2} \mathrm{O}\right)$ was added to the reaction mixture after the addition of monomers and the mixture was continuously stirred. After $1 \mathrm{~h}$, the reaction product was allowed to cool to ambient temperature and neutralized to $\mathrm{pH}$ 8 by addition of $1 \mathrm{~N}$ sodium hydroxide solution. The hydrogel was poured to excess non solvent ethanol $(200 \mathrm{~mL})$ and kept for $3 \mathrm{~h}$ to dewater. Then ethanol was decanted and the product scissored to small pieces. Again, $100 \mathrm{~mL}$ fresh ethanol was added and the hydrogel was kept for $24 \mathrm{~h}$. Finally, the filtered hydrogel is dried in oven at $60^{\circ} \mathrm{C}$ for $10 \mathrm{~h}$. After grinding using mortar, the powdered super absorbent was stored away from moisture, heat and light.

FTIR analysis: Fourier transform infrared (FTIR) spectra of samples were taken in $\mathrm{KBr}$ pellets, using an $\mathrm{ABB}$ Bomem MB-100 FTIR spectrophotometer (Quebec, Canada), at room temperature.

Surface morphology: The surface morphology of the gel was examined using scanning electron microscopy (SEM). After Soxhlet extraction with methanol for $24 \mathrm{~h}$ and drying in an oven, super absorbent powder was coated with a thin layer of gold and imaged in a SEM instrument (Leo, $1455 \mathrm{VP}$ ). Brunauer-Emmett-Teller (BET) analysis was used to determine the pore size of the hydrogels.

Thermogravimetric analysis: Thermogravimetric analyses (TGA) were performed on a Universal V4.1D TA Instruments (SDT Q 600) with 8-10 mg samples on a platinum pan under nitrogen atmosphere. Experiments were performed at a heating rate of $20{ }^{\circ} \mathrm{C} / \mathrm{min}$ until $600{ }^{\circ} \mathrm{C}$.

Swelling measurements: Hydrogel $(0.25 \mathrm{~g})$ were immersed in $250 \mathrm{~mL}$ solution with various $\mathrm{pH}$ values $(\mathrm{pH} 1.2$ and $\mathrm{pH}$ 7.4) at $37^{\circ} \mathrm{C}$ to reach swelling equilibrium. Swollen samples were then separated from unabsorbed water by filtering through a 100-mesh screen under gravity for $0.5 \mathrm{~h}$ without blotting the samples. The equilibrium swelling (ES) capacity in buffer solution was measured triple according to the following equation:

$$
\text { ES }(\mathrm{g} / \mathrm{g})=\frac{\text { Weight of swollen gel }- \text { Weight of dried gel }}{\text { Weight of dried gel }}
$$

The accuracy of the measurements was $\pm 3 \%$. The standard deviation ( $\mathrm{s}$ ) for a sample of data that is of limited size is given by the following equation:

$$
\mathrm{s}=\sqrt{\frac{\sum_{\mathrm{i}=1 \mathrm{Q}}^{\mathrm{N}}\left(\mathrm{X}_{\mathrm{i}}-\overline{\mathrm{X}}\right)^{2}}{\mathrm{~N}-1}}
$$

where $\left(X_{i}-X\right)$ is deviation from average of ith measurement and $\mathrm{N}$ is number of replicates of each measurement (here $\mathrm{N}=$ $3)$.

Encapsulation of model drug: Loading of ephedrine (20\% w/w, based on the total weight of the hydrogel) was carried out by swelling of dried polymeric hydrogel sample in phosphate buffer solution ( $\mathrm{pH} \mathrm{7.4)}$ at $37^{\circ} \mathrm{C}$. After immersing the vacuum dried powdered samples $(0.1 \mathrm{~g})$ for $24 \mathrm{~h}$, it was taken out, dried and accurately reweighed. The increase in the weight of the hydrogel was taken as the amount of drug loaded, ephedrine encapsulation efficiency percentage, (EE \%). The swollen hydrogels loaded with drug were placed in a vacuum oven, dried under vacuum at $37^{\circ} \mathrm{C}$ and stored until further investigation.

It should be pointed out that the phosphate may be lightly absorbed into the hydrogel along with the model drug. But the absorbed phosphate was very little. The anionic phosphate can't be largely absorbed by anionic hydrogel. However, we washed the loaded hydrogel by distilled water for removing the residues.

Spectrophotometric analysis of model drug: A UV/ visible spectrophotometer (Shimadzu, UV-2550) was used to determine the spectra of the drug. Model drug in aqueous solution was prepared for determining the maximum absorption wavelength. The absorbance value at the maximum wavelength of $276 \mathrm{~nm}$ of the model drug was read and the corresponding model drug concentrations were calculated from the calibration curve.

Determination of the amount of drug entrapped: The amount of ephedrine entrapped into the hydrogels was calculated by measuring the absorbance of the gelling medium at 276 $\mathrm{nm}$. The amount of ephedrine entrapped was estimated by the difference between the initial and the final amount of drug in gelling media. Encapsulation efficiency percentage was expressed as the weight of drug entrapped in the polymeric hydrogel divided by the initial weight of ephedrine in solution. Moreover, it is important to notice that the drug exhibited the same $\lambda_{\max }$ for whatever the release medium used in this study, as the free drug in water and the presence of dissolved polymers did not interfere with the absorbance of the drug at this wavelength.

Release studies: In vitro release studies were performed in simulated gastric fluid (SGF) and strongly in a simulated intestinal fluid (SIF) at $37^{\circ} \mathrm{C}$. Accurately weighed amounts of dried drug-loaded polymeric hydrogel (ranging from 0.1-0.2 g) were placed in beakers containing $1 \mathrm{~L}$ of the release medium at $37^{\circ} \mathrm{C}$. At periodic intervals $5 \mathrm{~mL}$ of aliquots were collected from the release medium and the ephedrine concentrations were measured using a spectrophotometer at $\lambda_{\max } 276 \mathrm{~nm}$. The percentage of cumulative amount of released ephedrine, obtained from three experiments, was calculated and plotted against time.

\section{RESULTS AND DISCUSSION}

FTIR spectroscopy: The grafting was confirmed by comparing the FTIR spectra of the collagen substrate with that of the grafted products. The band observed at $1644 \mathrm{~cm}^{-1}$ can be attributed to $\mathrm{C}=\mathrm{O}$ stretching in carboxamide functional groups 
of substrate backbone (Fig. 1a). The super absorbent hydrogel product comprises a collagen backbone with side chains that carry sodium carboxylate functional groups that are evidenced by peak at $1561 \mathrm{~cm}^{-1}$ (Fig. 1b). This characteristic band is due to asymmetric stretching in carboxylate anion that is reconfirmed by another peak at $1422 \mathrm{~cm}^{-1}$ which is related to the symmetric stretching mode of the carboxylate anion.

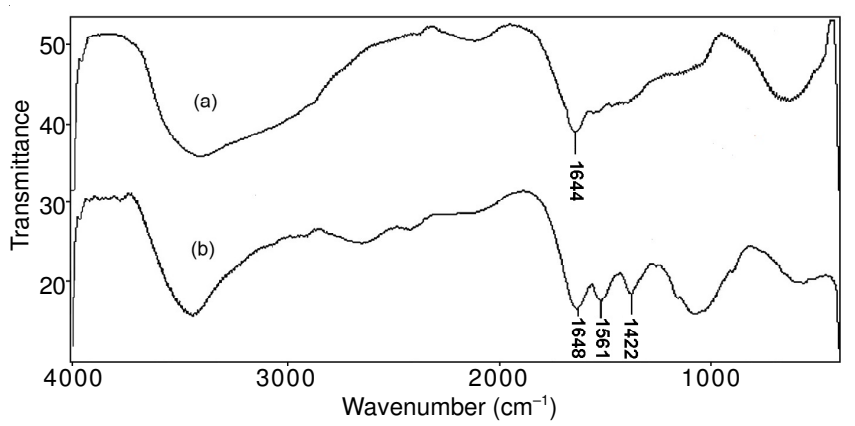

Fig. 1. FTIR spectra of collagen (a) and collagen-g-poly(acrylic acid-coitaconic acid) hydrogel (b)

Morphology of hydrogel: The morphology of the crosslinked hydrogel was observed by scanning electron microscope. Although the water inside the hydrogel was sublimed to make cavities, the structure of the hydrogel was preserved. Fig. 2 shows the scanning electron microscope photographs of the surface (Fig. 2a) and the cross-sectional area (Fig. 2b) of the hydrogel with interconnected pores. The hydrogel has a porous structure. It is supposed that these pores are the regions of water permeation and interaction sites of external stimuli with the hydrophilic groups of the graft copolymers. The cross-sectional view of hydrogels (Fig. 2b) also exhibited large, open, channel-like structure.
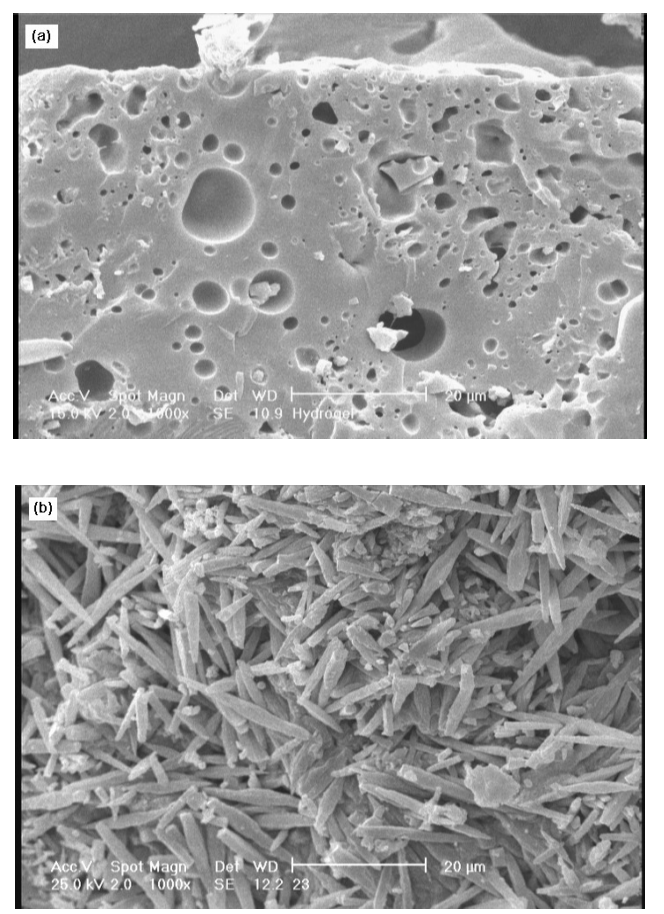

Fig. 2. SEM photograph of the hydrogel. (A) Surface of porous hydrogel; (B) Cross-sectional area of porous hydrogel. Surfaces were taken at a magnification of 1000 and the scale bar is $20 \mu \mathrm{m}$
The results of BET analysis showed that the average pore diameter of the synthesized hydrogel was $7.4 \mathrm{~nm}$. This proves the structure of the hydrogel is very porous and it could help to form a high-water-content hydrogel, as is generally shown in other hydrogels ${ }^{8}$.

Thermogravimetric analysis: TGA curves for collagen and collagen-based hydrogel are shown in Fig. 3. The grafted collagen has shown improvement in thermal stability as clear from TGA curve. The initial decomposition temperature of the collagen on grafting was increased from $168-402{ }^{\circ} \mathrm{C}$ with maximum decomposition rate at $523{ }^{\circ} \mathrm{C}$, in comparison to original decomposition temperature of $325^{\circ} \mathrm{C}$ of collagen. These observations have clearly indicated that grafting of poly(AA-co-IA) has improved the thermal stability of collagen.
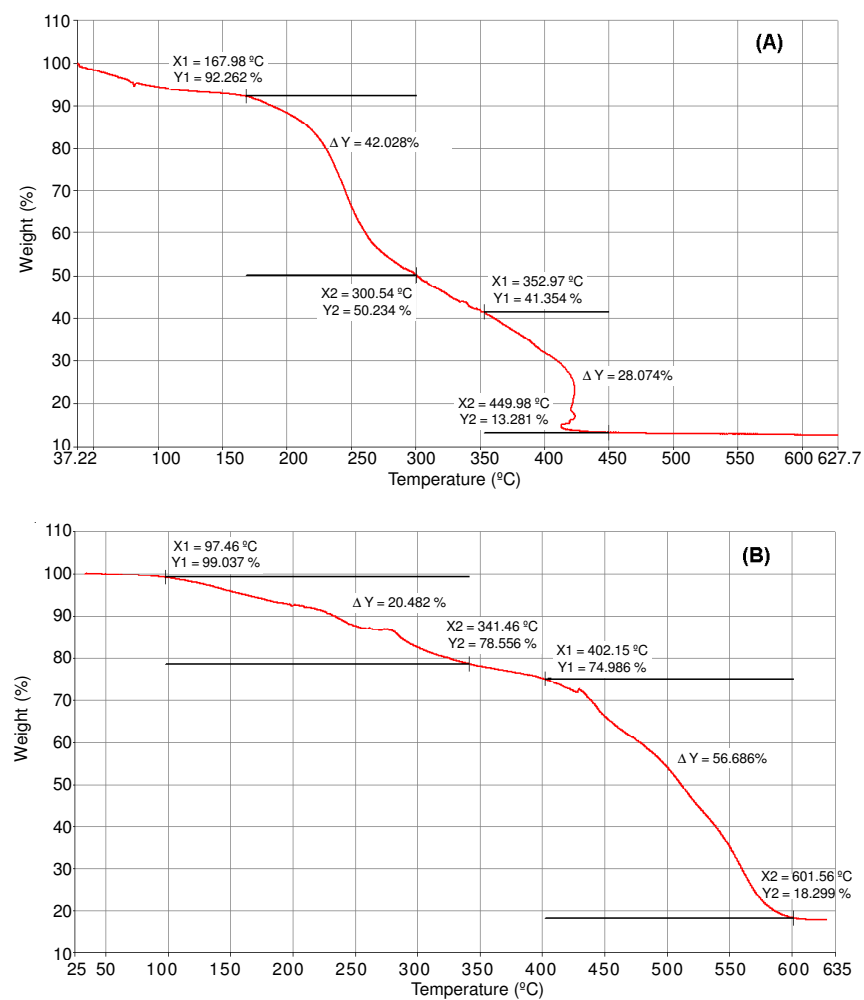

Fig. 3. TGA thermograms of (A) collagen and (B) collagen-g-poly(AAco-IA) super absorbent. Heating rate $20{ }^{\circ} \mathrm{C} / \mathrm{min}$, under $\mathrm{N}_{2}$

Effect of $\mathbf{p H}$ on equilibrium swelling: Since the $\mathrm{H}-$ collagen-g-poly(AA-co-AI) hydrogel (in the neutralization step) comprise anionic carboxylate groups, they exhibit sharp swelling changes at a wide range of $\mathrm{pHs}$. Therefore, the equilibrium swelling of H-collagen-g-poly(AA-co-AI) hydrogel was measured at various buffer solutions with $\mathrm{pH}$ ranged from 1-13 (Fig. 4). Under acidic pHs, most of the carboxylate anions are protonated, so the main anion-anion repulsive forces are eliminated and consequently swelling values are decreased. At higher pHs (5-8), some of carboxylate groups are ionized and the electrostatic repulsion between $\mathrm{COO}^{-}$groups causes an enhancement of the swelling capacity. Again, a charge screening effect of the counter ions (cations) limit the swelling at higher basic $\mathrm{pHs}$ ( $\mathrm{pHs}>8$ ).

pH-Responsiveness behaviour of the hydrolyzed hydrogel: The $\mathrm{pH}$-dependent swelling reversibility of the ionized hydrogel, H-collagen-g-poly(AA-co-AI), was examined 


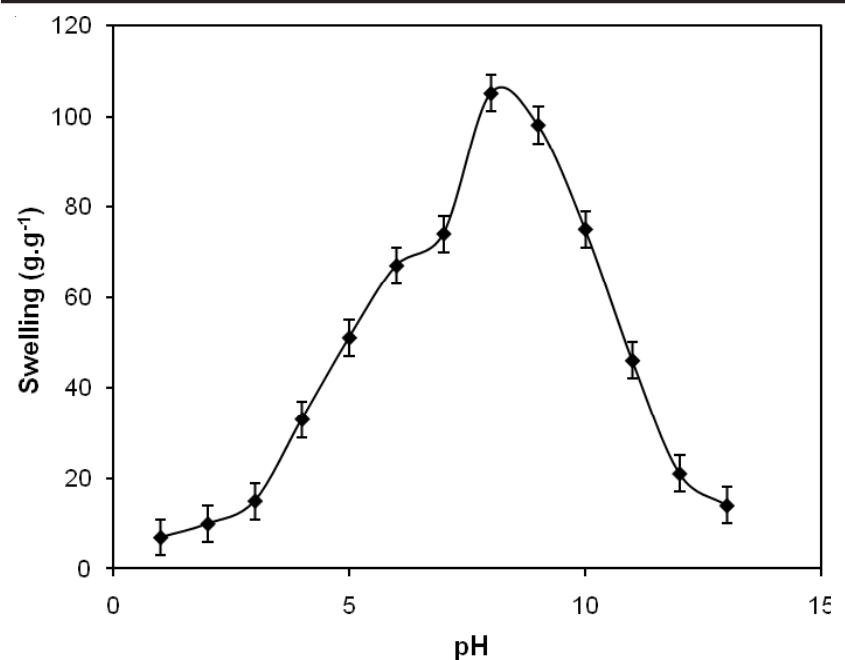

Fig. 4. Effect of $\mathrm{pH}$ of buffered solution on swelling of H-collagen-gpoly(AA-co-AI) super absorbent hydrogel

in two acidic and basic buffered solutions. Fig. 5 shows the reversible swelling-deswelling behaviour of the hydrogel at pHs 2 and 8. At $\mathrm{pH} \mathrm{8,} \mathrm{the} \mathrm{hydrogel} \mathrm{swells} \mathrm{due} \mathrm{to} \mathrm{anion-anion}$ repulsive electrostatic forces, while at $\mathrm{pH} 2$, it shrinks within a few minutes due to "screening effect" of excess cations. This sudden and sharp swelling-deswelling behaviour at different $\mathrm{pH}$ values makes the system to be highly $\mathrm{pH}$-responsive and consequently it may be a suitable candidate for designing controlled drug delivery systems. Similar swelling-pH dependencies have been reported in the case of other hydrogel systems $^{9-11}$.

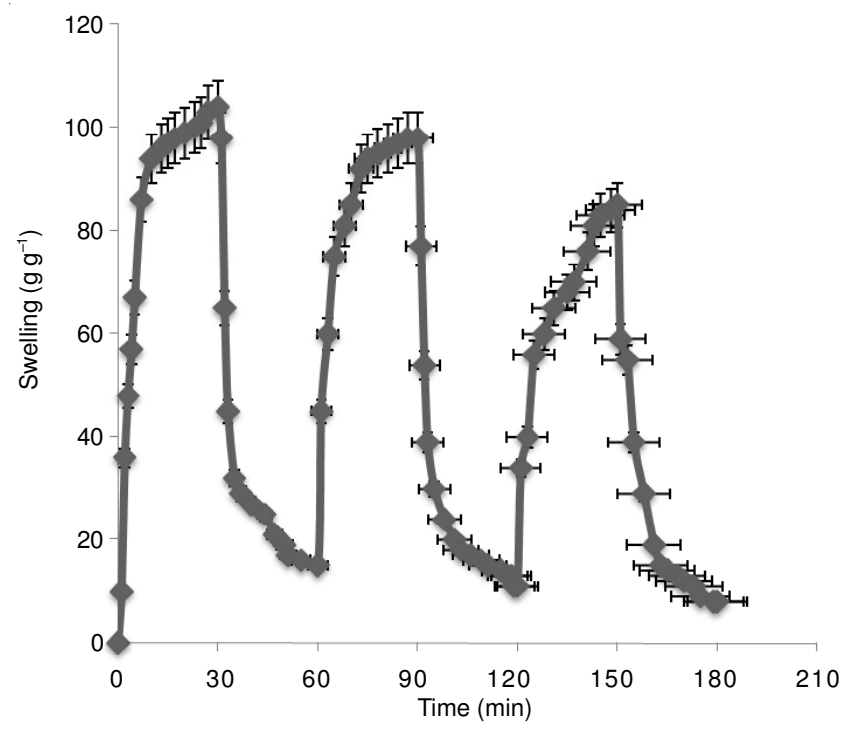

Fig. 5. On-off switching behaviour as reversible pulsatile swelling ( $\mathrm{pH} 8)$ and deswelling $(\mathrm{pH} \mathrm{2})$ of the H-collagen-g-poly(AA-co-AI) hydrogel. The time interval between the $\mathrm{pH}$ changes was $0.5 \mathrm{~h}$

\section{Conclusion}

A new $\mathrm{pH}$-responsive drug delivery system based on collagen hydrogel was developed for oral drug delivery of a poorly water-soluble drug to the intestinal environment. The super absorbent hydrogels exhibited high sensitivity to $\mathrm{pH}$, so that, several swelling changes of the hydrogel were observed in $\mathrm{pH}$ variations ( $\mathrm{pH} 1-13)$ of a wide range.

In comparison to other $\mathrm{pH}$-responsive natural-based hydrogel drug delivery system, our synthesized hydrogel has a good drug encapsulation and loading efficiency. The release value of drug from hydrogels at basic pHs was also considerable. Overall, the synthesized network is a novel hydrogel. The main application of this drug delivery system hydrogel is in vitro controlled release model drugs.

\section{REFERENCES}

1. B. Marandi, G. Mahdavinia and G.R. Ghafary, J. Appl. Polym. Sci., 120, 1170 (2011)

2. M.C. Branco, D.J. Pochan, N.J. Wagner and J.P. Schneider, Biomaterials, 31, 9527 (2010).

3. F.L. Buchholz and A.T. Graham, Modern Superabsorbent Polymer Technology, New York: Wiley (1997).

4. H. Cheng, J.L. Zhu, Y.X. Sun, S.X. Cheng, X.Z. Zhang and R.X. Zhuo, Bioconjug. Chem., 19, 1368 (2008).

5. L.Y. Chu, J.W. Kim, R.K. Shah and D.A. Weitz, Adv. Funct. Mater., 17, 3499 (2007).

6. L.Y. Chu, T. Yamaguchi and S.A. Nakao, Adv. Mater., 14, 386 (2002).

7. V. Crescenzi, L. Cornelio, C. Di Meo, S. Nardecchia and R. Lamanna, Biomacromolecules, 8, 1844 (2007).

8. D.T. Eddington and D.J. Beebe, Adv. Drug Deliv. Rev., 56, 199 (2004).

9. M. Hamidi, A. Azadi and P. Rafiei, Adv. Drug Deliv. Rev., 60, 1638 (2008).

10. S. Hua and A. Wang, Carbohydr. Polym., 75, 79 (2009).

11. S. Kakinoki, T. Taguchi, H. Saito, J. Tanaka and T. Tateishi, Eur. J. Pharm. Biol., 66, 383 (2007). 